\title{
Analysis On Deep Learning Approaches For Timely Detection Of Osteoarthritis
}

\author{
R Kanthavel $^{\mathrm{a}}$, R Dhaya ${ }^{\mathrm{b}}$, Harun Bangali $^{\mathrm{c}}$ \\ ${ }^{\text {a,c }}$ Department of Computer Engineering, College of Computer Science, King Khalid University, Abha, Saudi Arabia \\ ${ }^{\mathbf{b}}$ Department of Computer Science, College of Arts and Science-Sarat Abidha ,King Khalid University, Abha, Saudi Arabia \\ akanthavel2005@gmail.com, ${ }^{\mathrm{b}}$ dhayavel2005@gmail.com
}

Article History: Received: 10 November 2020; Revised 12 January 2021 Accepted: 27 January 2021; Published online: 5 April 2021

\begin{abstract}
Osteoarthritis is mainly a familiar kind of arthritis when an elastic tissue named Cartilage that softens the tops of the bones, cracks down. The Person with osteoarthritis can encompass joint pain, inflexibility, or inflammation and there is no particular examination for osteoarthritis and physicians take the amalgamation of both medical cum clinical record and X-rays imaging analysis to make a diagnosis of the state. Osteoarthritis is generally only detected following ache and bone scratch and in advance, analysis could permit for ultimate involvement to avoid cartilage worsening and bone injury. Through machinelearning algorithms, the system can be trained to automatically distinguish among people who would develop osteoarthritis and persons who would not with the detection of exact biochemical variances in the midpoint of the knee's cartilage. The outcome of the Machine learning Techniques will give the persons who are pre-symptomatic by the occasion of the baseline imaging and also the reduction in liquid concentration. In this study, we present the analysis of various deep learning techniques for timely detection of osteoarthritis disease. Several subsets of machine learning called deep learning techniques have been in use for the timely detection of osteoarthritis disease; and therefore analysis is needed highly to choose the best as far as accuracy and reliability are concerned.
\end{abstract}

Keywords: Osteoarthritis, arthritis, machine learning, Deep Learning techniques, imaging techniques

\section{Introduction}

A Deep mechanism to knowledge deliberates on the significance of what is well-read and that attention may possibly engage difficult the objects in opposition to a common acquaintance, daily understanding, and facts from further fields or paths[1]. In deep learning, the back propagation or back-prop technique is mentioned as the essential method for neural networks to be trained concerning whichever mistake in data calculation. Since no blood test is needed for the analysis of osteoarthritis but to keep out diseases that can origin secondary osteoarthritis and X-rays of the affected joints have been the major method osteoarthritis is recognized. MRI is used to evaluate numerous structural modifications connected to knee OA (KOA) along with Software image analysis methods to offer intention entirely quantitative dimensions of these amendments but for these methods human intervention is needed to analyze the same[2]. On the other hand, the deep learning process involves statistical machine learning, presents the possibility for improved automation to reduce reader occasion considerably.

The DL method can make a next remedy precise technique to review BML volume that suggests saving considerable time. The classification of KOA patients and harshness, a machine learning algorithm has been applied. The Support Vector Machine (SVM) belongs to a supervised learning form intended to discover a support vector that is used for Classification as well as Regression problems[4].

The presentation of strategic relapse investigation with the customary clinical measurable techniques and AI in ordering KOA patients and typical subjects is to be contemplated. The elements like age, sex, stature, weight, span of one step cycle, walk speed, rhythm, and the greatest knee flexion point should be utilized as covariates and the presence of (KOA patients bunch versus typical subjects gathering) to be utilize d as destitute variable in the strategic relapse model. The significant work in building disarray lattice with real and anticipated qualities, and pictured the exhibition of AI based characterization to order the seriousness of KOA patients by the step highlights [3]. The classifier exactness and the quantity of the highlights influence grouping a forward element determination calculation to be utilized will give thoughts regarding the choice of AI calculation. Past exactness results in the grouping of seriousness of knee osteoarthritis utilizing SVM and SVM with highlight determination calculation classifier have been analyzed regarding computing precision, explicitness, and sensitivity [5]. In the current day, practically every component of our regular day to day existence is overstated by AI and huge information. In like manner, clinicians can encounter distinctive through 
enormous information and AI by concerning AI to the clinical circumstances because of determination will turn out to be more reliable and accurate [6].

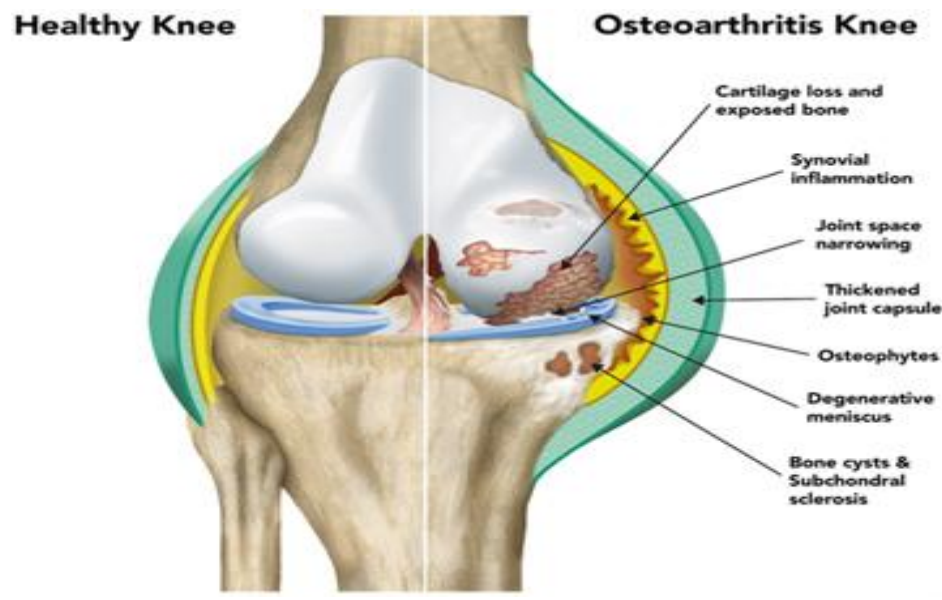

Figure 1: key structure skin texture of knee osteoarthritis

Figure 1 shows the key structure skin texture of knee osteoarthritis. The left elevation of the image demonstrates the ordinary knee and the right elevation illustrates the contaminated joint. There have been many deep learning methods that can contribute well to the KOA diagnosis accurately as a part of early detection. Among them, Reinforcement learning models are in great use[7]. This paper is arranged with three main sections namely, literature survey, Analysis of the Deep learning methods and their role in the detection of KOA, comparative analysis, and discussion.

\section{Preliminary Studies}

This section of this paper explains the literature survey on knee osteoarthritis and the various techniques in machine learning techniques. The merits and demerits of every deep learning technique also have been studied.

Tiulpin et al (2019)presented a multi-modal machine learning-based OA progression prediction framework that exploits rare radiographic records, as a medical analysis consequence and proceeding past the health condition of the patient. They also authorized their method on a self-governing examination customary of knee images by associating logistic regression technique to considerably expand the subject choice development for OA drugdevelopment hearings in serving the growth of tailored therapeutic tactics. In their report (2011), the authors executed ML algorithms to automatically measure knee osteoarthritis harshness from X-ray images rendering to the Kellgren \& Lawrence (KL) grades to estimate the enactment of numerous ML models like transfer learning, support vector machines and, fully connected neural networks based on their classification accuracy. They also instigated the undertaking of a spontaneous citation of the knee-joint region from the X-ray images and enumerating their sternness by preparing an earlier region CNN. Jihye Lim et al (2019) studied the issues with PCA analysis to make types from the patients' modest contextual health proceedings and recognized the incidence of osteoarthritis. Their tests indicated that the proposed method using deep the neural network.

Gan, HS et al (2020) reviewed to deliver an in-depth insight approximately a comprehensive group of traditional and deep learning division methods used in knee osteoarthritis investigation. Further, they composed convenient DL examinations to help as a basis of orientation to comfort the impending growth of DL prototypes. Almajalid et al (2019) adapted the U-net model to classify the bone structure on 3D knee MRI, which is an arrangement of numerous 2D portions. Ambellan F et al (2019) offered a technique for the automatic segmentation of knee bones and cartilage from magnetic resonance imaging (MRI) that chain a priori information of anatomical figure with CNN's by integrating 3D Statistical Shape Models (SSMs) to attain a healthy and precise segmentation of extremely knee structures.

Antony $\mathbf{J}$ et al (2017) presented a novel method to spontaneously measure the harshness of KOA using X-ray images using automatic localization and classifying the localized knee joint images utilizing completely CNN. Aprovitola A et al (2016) studied knee bone segmentation approaches from MR images by categorizing as per a priori information, the smooth computerization and operator communication. Ashinsky BG et al (2015) appraised the capability of ML to distinguish among MRI of standard and pathological human articular cartilage to progress a numerous linear least-squares regression model for arrangement and calculation of KOA. Ashinsky BG et al (2017) evaluated the capability of a ML algorithm to categorize in vivo MRI of human articular cartilage for OA. Bae KT et al (2009) established a semi-automated technique centered on a graph-cuts algorithm for segmentation and volumetric measurements of the cartilage from high-resolution knee MR images from the OAI Initiative 
database. Bourgeat P et al (2007) measured the automatic classification of textured tissues in 3D MRI to progress texture perception in the bone segmentation without the requirement of phase unwrapping.

Brem MH et al (2009) defined an assessment of semi-automated cartilage segmentation software to measuring balancing descriptions for possible exploit in longitudinal readings of knee OA. Cashman PMM et al (2002) clarified computerized strategies for the representation and diagramming of articular ligament in MR pictures of the osteoarthritis knee. Hang GH et al (2018) found the possibilities of an ML approaches to differentiate knees with pain from those without it in order to classify the structural types by creating a convolutional Siamese network to acquaintance MRI scans. Heng R et al (2020) examined possible instrumental issues of osteoarthritis, from MR images through mounting and authenticating an entirely automated DL model to fragment the patella and distal femur cortex. Dam E, Lillholm M etal (2015) projected a division model for completely programmed division of knee MRI to demonstrate precision. Dodin P et al (2015) pointed toward expanding a novel programmed division calculation for human knee ligament volume measurement from MRI by utilizing surface investigation techniques. Dodin P et al (2015) pointed toward mounting a completely robotized bone division strategy for the human knee from MR pictures. Gan H-S et al (2014) planned cooperative knee cartilage abstraction software to shield three important structures namely perception, quickness, and suitability by means of multi-label random walks procedure.From the literature survey, it is inferred that there have been several ML and Deep Learning algorithms used to detect the OA and KOA and hence a need has been raised to compare all the Deep learning algorithms in terms of Accuracy, specificity and, Sensitivity.

\section{Deep learning methods and their role in the detection of KOA}

There have been mainly three deep learning techniques used in the early detection of knee osteoarthritis namely Segmentation techniques, Feature Extraction Techniques and, classification techniques. The following is the extensive analysis of the above deep learning methods.[8]

\section{Segmentation techniques for KOA:}

Segmentation is the strategy of isolating or parceling a picture into parts, called sections. It is for the most part helpful for applications like picture pressure or article acknowledgment, because for these kinds of uses, it is wasteful to deal with the entire picture. Segmentation is the cycle partitioning a picture into districts with comparative properties; for example, dim level, shading, surface, brilliance, and differentiation [9]. The part of segmentation is to partition the items in a picture. Picture division incorporates changing over an image into a grouping of districts of pixels that are tended to by a cover or a named picture. By isolating a picture into sections, you can deal with just the significant portions of the picture as opposed to preparing the whole picture. The Edge Detection strategy is one of the division strategies. Figure 2 demonstrates the Edge Detection Methods used for Knee OA.

- $\quad$ Edge Detection technique: Edge detection technique is used for the examination of the ailment that joins Contrast redesign and thresholding for patella feature point extraction. In this edge detection procedure, Watershed, Otsu's and, discrete development inferences are used. Watchful edge count contrast improvement, histogram change, and thresholding are used on knee x-bar pictures. Edge detection system is used to choose the district of joint space in $\mathrm{OA}[10]$. In this procedure, we got half affectability, $100 \%$ distinction and, positive perceptive worth and, 91.84\% negative insightful worth. The Edge detection procedures like Sobel, Prewitt Robert, Zero cross and Canny edge strategies were used to imagine knee joint tendon. Watchful edge detection system works commendably with the given pictures when diverged from various procedures. Otsu's figuring, Vertical edge detection method, Automatic area detection system, Euclidian count and, related part estimation are used which came about the Cartilage thickness of knee was successfully finished [11]. 


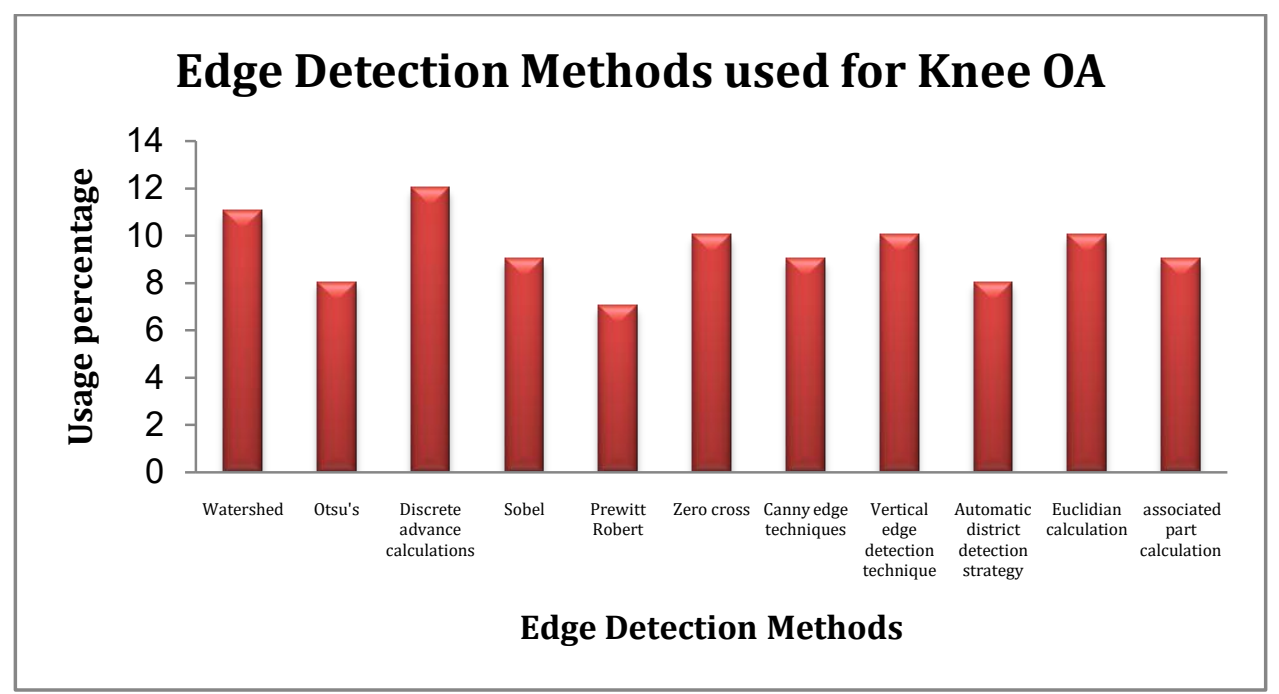

Figure 2: Edge Detection Methods used for Knee OA

\section{Feature Extraction Techniques:}

The primary goal of feature extraction is the programmed extraction of features from the contribution, to address it in a one- of -a - kind and minimal type of a solitary worth or lattice vector [12]. The three distinct methods of feature extraction are flat heading, vertical bearing and askew course. Recognition rate for vertical, even and, slanting-based feature extraction utilizing feed-forward back engendering neural organization as categorization stage. Figure 3 shows the Feature Extraction Techniques used for KOA.

- In Feature Extraction Techniques, the highlights figured were Zernike highlights, Multi - scale histograms, Tamura surface highlights, Haralick include, real highlights, Random change highlights, Chebyshev Statistics, Gabor channel, Multi-scale histograms, First 4 minutes, Edge bits of knowledge highlights, High Contrast includes, and Weighted Nearest Neighbor classifier. Fake Neural Networks and feature extraction methods are utilized to identify knee OA. Dark level Co-event Matrix textural features, Shape characteristics were processed [13]. X-ray characterization is proposed utilizing Pattern acknowledgment and staggered decline with the WND-CHRM technique. Surface features, factual features, Polynomial decay of crude pictures were processed. The stride design procedure is utilized for 3D pictures of knee OA. Features were figured utilizing GRF vectors and arranged to utilize the Nearest Neighbor rule. Quantitative surface examination utilizing MR pictures. Features figured were mean power, standard deviation, perfection, third second, consistency and, entropy[14].

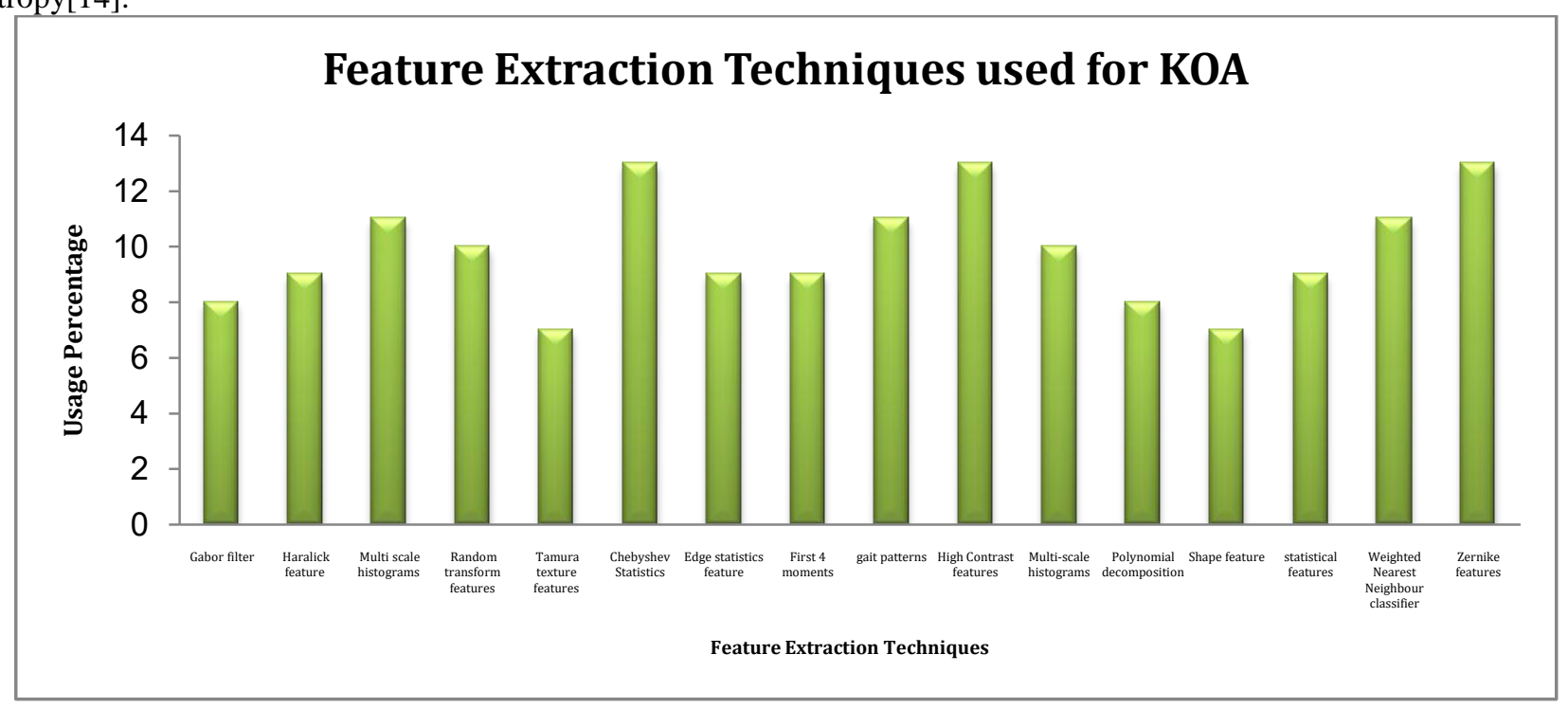

Figure 3 : Feature Extraction Techniques used for KOA

\section{Classification methods for KOA:}

The picture classification in clinical pictures incorporates shape highlights, shading histogram highlights, shading second highlights, and surface highlights. The majority of the past examinations utilize worldwide 
highlights to order clinical images. The primary classification strategies are supervised classification, unsupervised classification and semi-supervised classification. Classification of OA based on Local binary pattern (LBP) is used [15]. Euclidian, Cosine, Manhattan, correlation features using K-nearest neighbor classifier. Classification technique stands on ANN is used to measure the severity of Osteoarthritis disease [16]. Convolutional Neural network (CNN) based techniques are used for detecting and classification of Osteoarthritis. MRI categorization is proposed using Pattern recognition \& multilevel regression with WNDCHRM method. Figure 4 shows the Classification methods used for KOA.

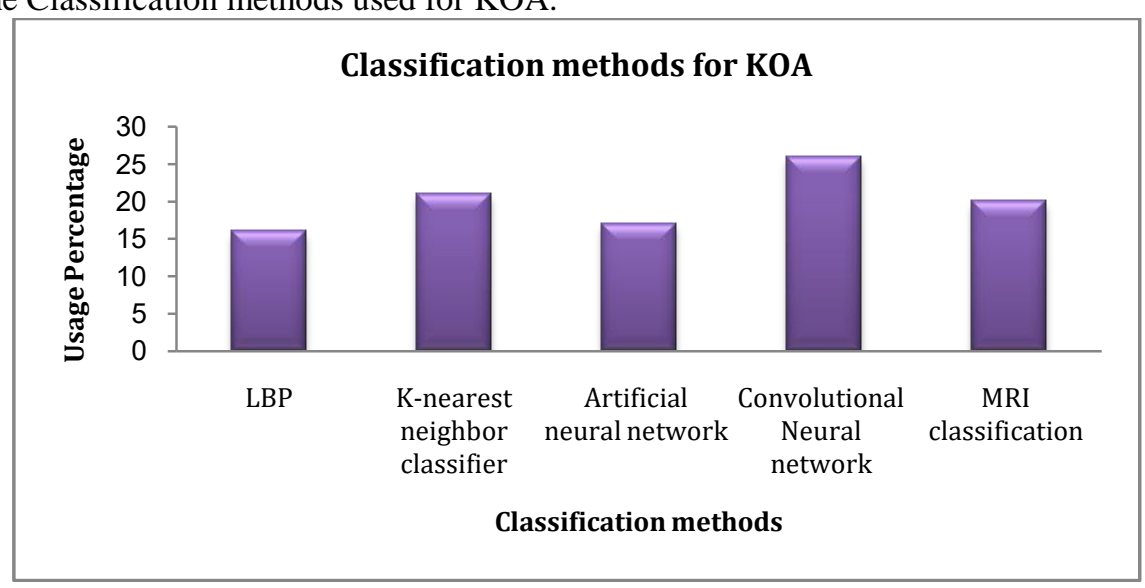

Figure 4: Classification methods for KOA

\section{Comparative studies of Reinforcement Learning}

The comparative analysis of the Deep Learning methods has been explained as follows in terms of testing time, training time, accuracy and, loss function. Here figure 4 shows the list of deep learning methods used for this paper.

Figure 4: list of deep learning methods used

VGG 16: In VGG-16, it suggests that there are 16 layers as the significance of the association arrangement. During readiness, the information picture is experienced a stack of convolutional layers, where we use channels with a little responsive field: 3 x 3 . The convolution layer limits are meant as open field size) - (number of channels)"[17]. For example, conv3-64 techniques open field size: 3 x 3 with 64 quantities of channels. At that point, in this work, spatial pooling is finished by five max-pooling layers, which follow a segment of the conv layers. Max pooling is performed over a 2 × 2 - pixel window. So how would we get an incentive for $\mathrm{z}(\mathrm{i}, \mathrm{j}, \mathrm{k})$ for a given line $\mathrm{I}$, section $\mathrm{j}$, and divert $\mathrm{k}$ in $\mathrm{z}$. we utilize the convolution work

$$
\mathrm{z}[1](\mathrm{i}, \mathrm{j}, \mathrm{k})=\sum_{\mathrm{l}=1}^{3} \sum_{\mathrm{m}=1}^{3} \sum_{\mathrm{n}=1}^{3} \mathrm{x}(\mathrm{i}+\mathrm{l}-1, \mathrm{j}+\mathrm{m}-1, \mathrm{n}) \mathrm{Wc}(1, \mathrm{~m}, \mathrm{n}, \mathrm{k})+\mathrm{b}(\mathrm{k}, 1)
$$

Resnet 50: Residual network engineering is propelled dependent on VGG design. This examination, contrasted the aftereffects of tests and the ResNet-50 technique[18]. ResNet-50 implies that this profound learning technique has 50 layers. As a rule, in profound convolutional neural networks, a few layers are stacked and prepared for the main job. The network learns a few low/medium/significant level highlights toward the finish of its layers. Residual can be perceived as a decrease in highlights gained from the information layer. ResNet does this by utilizing alternate route associations (which straightforwardly interface contribution from nth layer to a few (n + $\mathrm{x})_{\mathrm{th}}$ layers. Officially, let $\mathrm{H} \_1$ be the yield of the 1 -th residual square, $\mathrm{f}$ - 1 be the planning characterized by the 1 - ${ }^{\text {th }}$ square's weighted planning, b_l be a Bernoulli irregular variable that is just 1 or 0 (showing whether a square is dynamic), during preparing: 


$$
\mathrm{H}_{\mathrm{l}}=\operatorname{ReLU}\left(\mathrm{b}_{\mathrm{l}} * \mathrm{f}_{\mathrm{l}}\left(\mathrm{H}_{1-1}\right)+\mathrm{id}\left(\mathrm{H}_{\mathrm{l}-1}\right)\right)
$$

Dense Net (Densely Connected Convolutional Networks) 121: DenseNet is the advancement of ResNet. In DenseNet design, expressly recognizes data added to the organization and data that is held[19]. The DenseNet layer is thin; simply add a little arrangement of highlight guides to the "aggregate information" of the organization and save the excess guide highlights unaltered, and the last classifier settles on a choice dependent on all element maps in the organization. Thusly, the $1^{\text {th }}$ layer gets the component guides of every single going before layer, $\mathrm{x} 0, \ldots$ ., $\mathrm{xl}-1$, as information: $\mathrm{xl}=\mathrm{Hl}([\mathrm{x} 0, \mathrm{x} 1, \ldots, \mathrm{xl}-1])$, where $[\mathrm{x} 0, \mathrm{x} 1, \ldots, \mathrm{xl}-1]$ alludes to the connection of the element maps delivered in layers $0, \ldots, 1-1$. Due to its thick availability, we allude to this organization's engineering as Dense Convolutional Network (DenseNet). For simplicity of usage, we connect the numerous contributions of $\mathrm{Hl}(\bullet)$

CNN with LSTM:During the arrangement and testing stages, a couple of cycles were finished as follows: preprocessing is driven actually by managing the knee joint from $\mathrm{x}$-shaft pictures with estimations of 400x 100 pixels. By then, each managed picture is extended by transformation and stacked alongside the primary picture so CNN input is as sequential data. This system is done in light of the fact that LSTM is generally completed in back to back data. Moreover, in the CNN communication, we use three convolutions; each channel size is $3 \times 3$ and with the amount of channels 32, 64 and, 128. The conditions that portray the convolution layer are: $y_{j}^{(1)}=\left(\sum_{i \in c j} t\right.$ $\left.i^{1-1} \otimes w_{i j}^{(1)}\right)+b_{j}^{(1)}$

Where cj is a bunch of information includes diagrams. $\mathrm{b} 1 \mathrm{j}$ is the inclination, $\mathrm{y} \mathrm{l} \mathrm{j}$ the yield of the convolution and, $w(1)$ ij the convolution part. $t \mathrm{j} l=\mathrm{f}(\mathrm{y} j \mathrm{j}(\mathrm{l})) \mathrm{t} \mathrm{jl}$ the component diagram of the convolution layer $1 . \mathrm{f}(\mathrm{x})=$ $\max (0, \mathrm{x}) \mathrm{f}$ is known as the actuation work. In this work, we utilize an amended direct unit characterized in Equation

Deep Learning-based Method:A procedure using a straight SVM and the Sobel level picture tendencies as the features for distinguishing the knee joint core interests. The prominent Sobel edge revelation figuring uses the vertical and the level picture points. The motivation for this is that knee joint pictures basically contain even edges. The image patches $(20 \times 20$ pixels $)$ containing the knee joint center are taken as the positive planning tests and the image patches $(20 \times 20$ pixels $)$ notwithstanding the knee joint center are taken as the negative getting ready tests. In the wake of removing Sobel level slants for the positive and negative models, a straight SVM was readied. To recognize the knee joint concentration from both left and right knees, input pictures are part down the center to withdraw left and right knees freely. A sliding window $(20 \times 20$ pixels $)$ is used on either segment of the image, and the Sobel even point features are eliminated for each image fix. The image fix with the most extraordinary score reliant on the SVM .decision limit is recorded as the recognized knee joint center, and the zone ( $300 \times 300$ pixels) around the knee joint center is isolated from the information pictures using the relating recorded bearings We take a gander at the models using both mean squared misstep (MSE) and standard multiclass plan estimations. We decided the mean squared misstep using the standard condition

$$
\operatorname{MSE}=1 / \mathrm{n} \sum_{\mathrm{i}=1}^{\mathrm{n}}\left(\mathrm{yi}-\mathrm{y}^{\wedge} \mathrm{i}\right)
$$

Where $\mathrm{n}$ is the number of test tests, yi is the valid (whole number) name and yi is the anticipated name. For the order network, the anticipated marks yi are whole numbers and for the relapse network they are genuine number

Aprofound neural Network-based Statistical Data: Here four terms are used as, i.e., certifiable positives (GP), real negatives $(\mathrm{GN})$, fake positives $(\mathrm{BP})$, and false negatives $(\mathrm{BN})$, to handle the introduction limits and evaluate the made models. GP was the adequately foreseen OA, and GN was the precisely foreseen non-OA. BP and BN were the incorrectly foreseen OA and non-OA, independently. For the most part, the display of recognizable proof or assumption computations is assessed by the Accuracy. Nevertheless, the two-classed educational file we used in this assessment was imbalanced. In the educational files, there was more non-osteoarthritis data than osteoarthritis data (1240). To address this class cumbersomeness issue, we used three additional estimations for real appraisal: affectability (Sn), distinction (Sp), and positive-farsighted worth (PPV). Affectability is a probability of the foreseen cases of osteoarthritis status, distinction is a probability of the foreseen occasions of non-osteoarthritis status, and positive farsighted worth is a probability of the cured assumption for the osteoarthritis status.

Deep Neural network with a novel ordinal loss:first devise a customizable ordinal grid $\mathrm{W}$ to indicate the punishment loads between the anticipated evaluation and the genuine evaluation. $\mathrm{W}$ is an $\mathrm{n} * \mathrm{n}$ square lattice, where wi, $\mathrm{j} \in \mathrm{W}$ represents the punishment weight of foreseeing grade $\mathrm{j}$ to review $\mathrm{I}$, here $\mathrm{I}, \mathrm{j} \in\{0,1, \ldots, \mathrm{n}-1\}$ and $n=5$ in the knee KL evaluating task. The network on base left of w:, $m$ addresses the discipline vector of assessment $\mathrm{m}$. We set the discipline weight of every assessment to itself fixed as 1 and to the rest grades to be higher if the rest grades are far off. Considering this ordinal grid and the yield probabilities by the softmax layer, the proposed ordinal mishap is portrayed as: 
Loss $=\quad \sum_{\mathrm{i}=0}{ }^{\mathrm{n}-1}$ wi, $\mathrm{m} \cdot \mathrm{qi}$

Where $\mathrm{m}$ is the genuine KL evaluation of the info picture; $q \mathrm{i}=$ pi on the off chance that $\mathrm{I}=/ \mathrm{m}$, in any case, qi = 1 - pi. Limiting misfortune in Eq. (1) expects pm to be near 1.0 and distant evaluation to have considerably more modest likelihood because of its relating high punishment weight.



Figure 5: Knee OA Severity Classification Results- Training Time

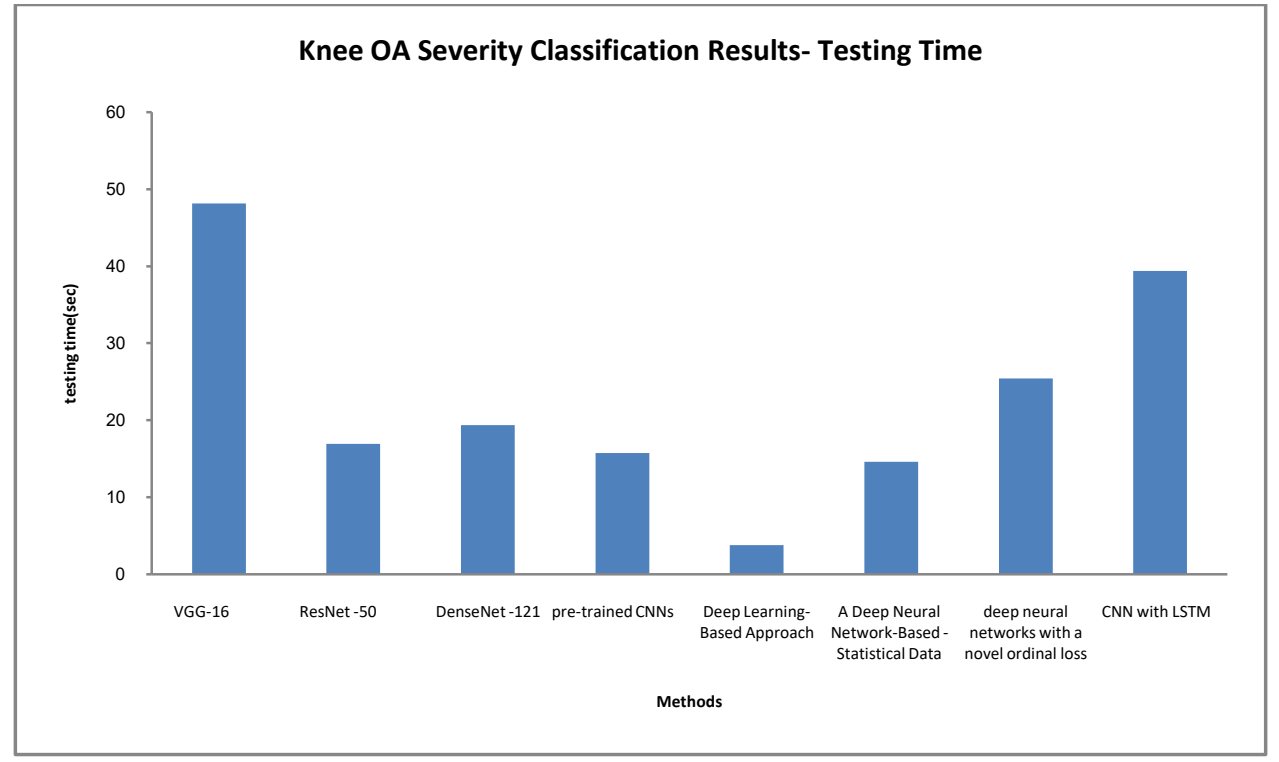

Figure 6: Knee OA Severity Classification Results- Testing Time

Training time/Testing time is a strategy to quantify the precision of the model of Knee OA. . It is called Training time/Test time since you crack the informational collection into two sets: a preparation set and a testing set. $4 / 5^{\text {th }}$ for preparing and $1 / 5^{\text {th }}$ for testing. You train the model using the preparation set. In a dataset, a preparation set is completed to build up a model, while a test (or endorsement) set is to support the model manufactured. ... Consequently, we use the preparation data to fit the model and testing data to test it. The models produced are to foresee the outcomes obscure which is named as the test set. Figure 5 and 6 shows the training time of Knee OA Severity Classification outcomes and Testing time of Knee OA Severity Classification outcomes. In the above 2 diagrams, it clearly shows that a variety of models are taken and the testing results depend on the various methods and training data seta are used. 


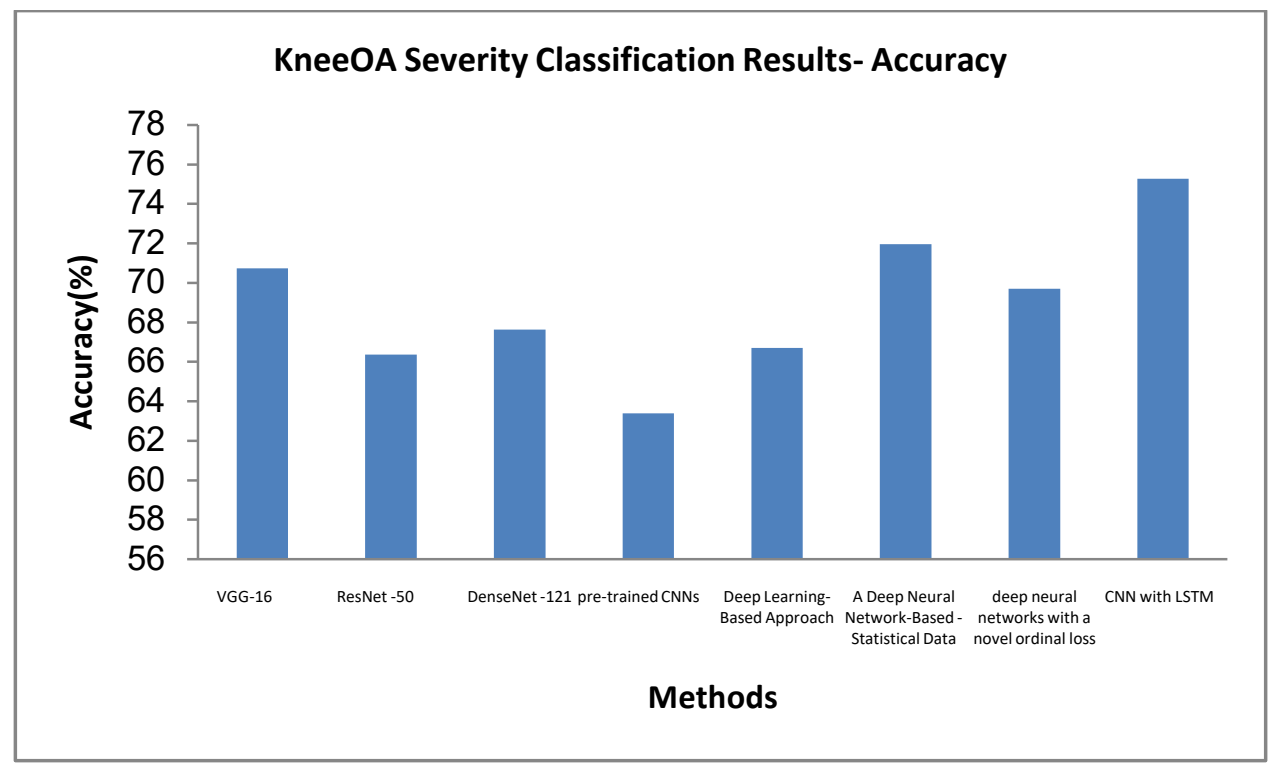

Figure 6: Knee OA Severity Classification Results- Accuracy

Figure 7 shows the accuracy of the Knee OA Severity Classification Results. In the projected technique, the grouping of knee OA seriousness, specifically KL rating is gotten dependent on removal of highlights from xbeam pictures, and rates correctness is utilized as a presentation measures. Accurateness outcomes are acquired for every evaluation, where the evaluations demonstrate the seriousness of knee OA from the given techniques that appeared in the chart. Accuracy grade is the accurate estimation of all evaluation. Each evaluation is tried into a model/strategy that has been assembled. Normal accurateness by grade is the normal of accurateness of every evaluation.

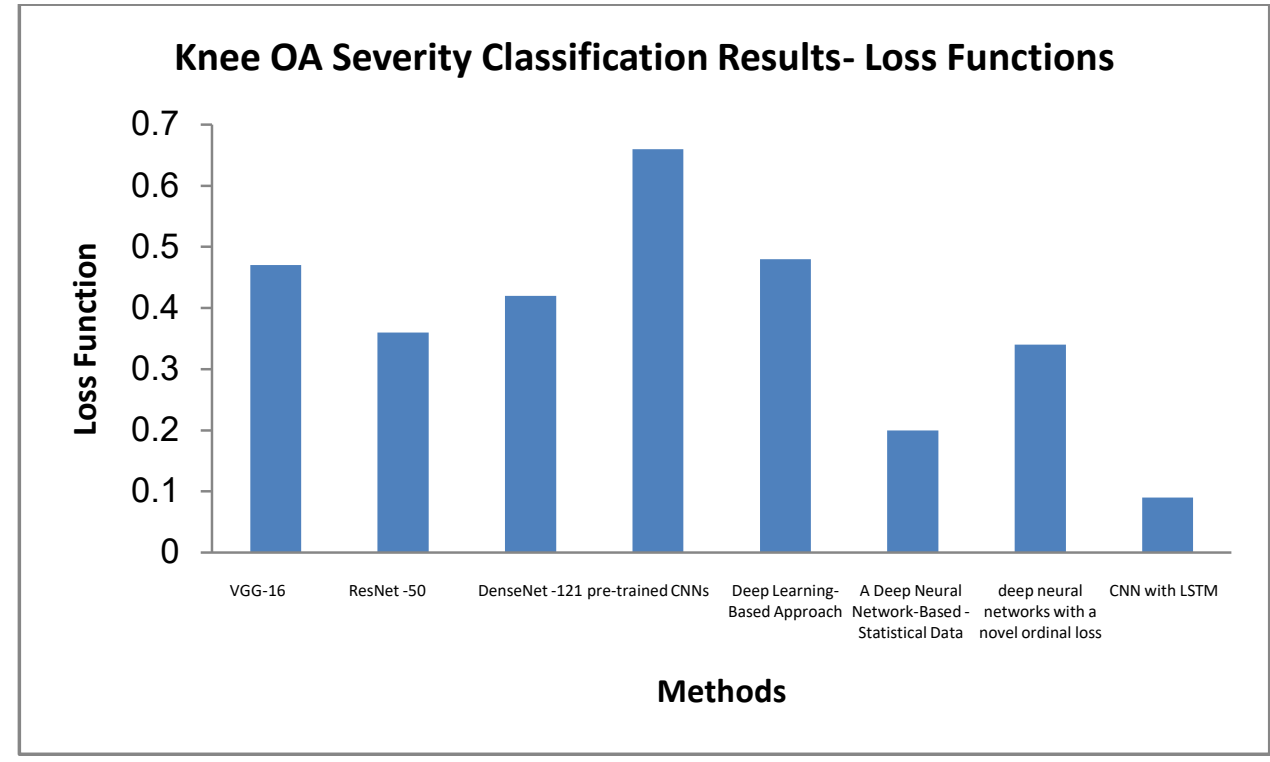

Figure 8: Knee OA Severity Classification Results- Loss Functions

Figure 8 shows the Knee OA Severity Classification Results-Loss Functions. Every cover has straight admittance to the angle of the first loss capacity and information indicator, which prompts implied top to bottom management. This assists preparing further organization engineering. Besides, we likewise see that strong associations have a regularization impact, which lessens over fitting on assignments with a more modest preparing set size. Here in this figure, a few strategies are utilized for accomplishing the loss capacity of knee OA Severity Classification.

\section{DISCUSSION}

As of now, as doctors have impelled to be acquainted with KOA that is started by an assortment of highlights along with inherited, provocative, and programmed measures, to a firm sum, is viewed as an avoidable illness. 
Hence, early inclusion is essential precisely for the early investigation of KOA. Despite the fact that there are conceivable outcomes to accomplish considerable consistency in the AI based grouping of KOA patients. Be that as it may, the modification of sporadic stride model or future treatment is yet to be underlying.

\section{CONCLUSION}

This paper presented the depth analysis of various Deep learning methods and their role in the detection of KOA and comparison studies. From this study, KOA classification strategy utilizing ML for the KOA patients and typical subjects has been examined and contrasted execution and the ordinary measurable preparing method. It is perceived that the AI based strategy could supplement traditional calculated relapse examination in the characterization of KOA patients and normal subjects. In addition, the AI method is viewed as ready to win over a clinician's be shy of induction inferable from limits on the intricacy and tremendous amount of information possible in stride examination. Additionally, it is normal that the aftereffects of this learning can be valuable for the analysis of KOA patients and rehabilitative involvement all the way through gait improvement.

\section{Acknowledgement:}

We would like to thank King Khalid University for funding this work through General Research Projectunder Grant Number GRP / 119/42 .We thank "anonymous" reviewers for their so-called insights.

Conflicts of Interest: The authors declare no conflict of interest..

\section{References}

Tiulpin, A., Klein, S., Bierma -Zeinstra, S.M.A. et al. Multimodal Machine Learning-based Knee Osteoarthritis Progression Prediction from Plain Radiographs and Clinical Data. Sci Rep 9, 20038 (2019). https://doi.org/10.1038/s41598-019-56527-3

Automatically Quantifying Radiographic Knee Osteoarthritis Severity Final Report - CS 229 - Machine Learning

JihyeLim ,Jungyoon Kim and SongheeCheon,” A Deep Neural Network-Based Method for Early Detection of Osteoarthritis Using Statistical Data”, Int J Environ Res Public Health . 2019 Apr 10;16(7):1281. doi: 10.3390/ijerph16071281.

Gan, HS.,Ramlee, M.H., Wahab, A.A. et al. From classical to deep learning: review on cartilage and bone segmentation techniques in knee osteoarthritis research. ArtifIntell Rev (2020). https://doi.org/10.1007/s10462-020-09924-4

Almajalid R, Shan J, Zhang M, Stonis G, Zhang M (2019b) Knee bone segmentation on three-dimensional MRI. In: IEEE 18th international conference on machine learning and applications (ICMLA), 16-19 Dec. 2019, pp 1725-1730. https://doi.org/10.1109/ICMLA.2019.00280

Ambellan F, Tack A, Ehlke M, Zachow S (2019) Automated segmentation of knee bone and cartilage combining statistical shape knowledge and convolutional neural networks: data from the osteoarthritis initiative. Med Image Anal 52:109-118. https://doi.org/10.1016/j.media.2018.11.009

Antony J, McGuinness K, Moran K, O’Connor N Automatic detection of knee joints and quantification of knee osteoarthritis severity using convolutional neural networks. In: International conference on machine learning and data mining in pattern recognition, 2017. Lecture Notes in Computer Science. Springer, Cham. https://doi.org/10.1007/978-3-319-62416-7_27

Aprovitola A, Gallo L (2016) Knee bone segmentation from MRI: a classification and literature review. Biocybern Biomed Eng 36:437-449. https://doi.org/10.1016/j.bbe.2015.12.007

Ashinsky BG et al (2015) Machine learning classification of OARSI-scored human articular cartilage using magnetic resonance imaging. OsteoarthrCartil 23:1704-1712. https://doi.org/10.1016/j.joca.2015.05.028

Ashinsky BG et al (2017) Predicting early symptomatic osteoarthritis in the human knee using machine learning classification of magnetic resonance images from the osteoarthritis initiative. J Orthop Res 35:2243-2250. https://doi.org/10.1002/jor.23519

Bae KT, Shim H, Tao C, Chang S, Wang JH, Boudreau R, Kwoh CK (2009) Intra- and inter-observer reproducibility of volume measurement of knee cartilage segmented from the OAI MR image set using a novel semi-automated segmentation method. OsteoarthrCartil 17:1589-1597. https://doi.org/10.1016/j.joca.2009.06.003

Bourgeat P, Fripp J, Stanwell P, Ramadan S, Ourselin S (2007) MR image segmentation of the knee bone using phase information. Med Image Anal 11:325-335. https://doi.org/10.1016/j.media.2007.03.003

Brem MH et al (2009) Magnetic resonance image segmentation using semi-automated software for quantification of knee articular cartilage-initial evaluation of a technique for paired scans. SkeletRadiol 38:505-511. https://doi.org/10.1007/s00256-009-0658-1

Cashman PMM, Kitney RI, Gariba MA, Carter ME (2002) Automated procedures for visualization and mapping of articular cartilage in MR images of the osteoarthritic knee: a base technique for the assessment of 
microdamage and submicro damage. IEEE Trans Nanobiosci 99:42-51. https://doi.org/10.1109/TNB.2002.806916

hang GH, Felson DT, Qiu S, Capellini TD, Kolachalama VB (2018) Predicting bilateral knee pain from MR imaging using deep neural networks. bioRxiv:463497 https://doi.org/10.1101/463497

Heng R et al (2020) Fully automated patellofemoral MRI segmentation using holistically nested networks: implications for evaluating patellofemoral osteoarthritis, pain, injury, pathology, and adolescent development. MagnReson Med 83:139-153.

Dam E, Lillholm M, Marques J, Nielsen M (2015) Automatic segmentation of high- and low-field knee MRIs using knee image quantification with data from the osteoarthritis initiative. J Med Imaging 2:024001

Dodin P, Pelletier J, Martel-Pelletier J, Abram F (2010) Automatic human knee cartilage segmentation from 3-D magnetic resonance images. IEEE Trans Biomed Eng 57:2699-2711. https://doi.org/10.1109/TBME.2010.2058112

Dodin P, Martel-Pelletier J, Pelletier J-P, Abram F (2011) A fully automated human knee 3D MRI bone segmentation using the ray casting technique. Med BiolEngComput 49:1413-1424. https://doi.org/10.1007/s11517-011-0838-8

Gan H-S, Tan T-S, Wong L-X, Tham W-K, Sayuti KA, Abdul Karim AH, bin Abdul Kadir MR (2014a) Interactive knee cartilage extraction using efficient segmentation software: data from the osteoarthritis initiative. Bio-Med Mater Eng 24:3145-3157.

J. Antony, K. McGuinness, K. Moran, and N. E. O'Connor, "Automatic Detection of Knee Joints and Quantification of Knee Osteoarthritis Severity Using Convolutional Neural Networks,”MLDM 2017, vol. LNAI 10358, pp. 376-390, 2017.

J. Lim, J. Kim, and S. Cheon, “A Deep Neural Network-Based Method for Early Detection of Osteoarthritis Using Statistical Data,” Int. J. Environ. Res. Public Health, vol. 16, no. 7, p. 1281, 2019.

A. Tiulpin, J. Thevenot, E. Rahtu, P. Lehenkari, and S. Saarakkala, "Automatic knee osteoarthritis diagnosis from plain radiographs: A deep learning-based approach,” Sci. Rep., vol. 8, no. 1, pp. 1-10, 2018 